ARTICLE

Received 8 Jan 2013 | Accepted 13 May 2013 | Published 7 Jun $2013 \quad$ DOl: 10.1038/ncomms2994

\title{
Brillouin cavity optomechanics with microfluidic devices
}

Gaurav Bahl', Kyu Hyun Kim², Wonsuk Lee 2,3 , Jing Liu ${ }^{3}$, Xudong Fan³ \& Tal Carmon²

Cavity optomechanics allows the parametric coupling of phonon- and photon-modes in microresonators and is presently investigated in a broad variety of solid-state systems. Optomechanics with superfluids has been proposed as a path towards ultra-low optical- and mechanical-dissipation. However, there have been no optomechanics experiments reported with non-solid phases of matter. Direct liquid immersion of optomechanics experiments is challenging, as the acoustic energy simply leaks out to the higher-impedance liquid surrounding the device. Here we confine liquids within hollow resonators to circumvent this issue and to enable optical excitation of mechanical whispering-gallery modes at frequencies ranging from 2 to $11,000 \mathrm{MHz}$. Our device enables optomechanical investigation with liquids, while light is conventionally coupled from the outer dry side of the capillary, and liquids are provided by means of a standard microfluidic inlet.

\footnotetext{
${ }^{1}$ Mechanical Science and Engineering, University of Illinois at Urbana-Champaign, 1206 West Green Street, Urbana, Illinois 61801, USA. ${ }^{2}$ Electrical Engineering and Computer Science, University of Michigan, 2200 Bonisteel Boulevard, Ann Arbor, Michigan 48109, USA. ${ }^{3}$ Biomedical Engineering, University of Michigan, 1101 Beal Avenue, Ann Arbor, Michigan 48109, USA. Correspondence and requests for materials should be addressed to G.B.

(email: bahl@illinois.edu).
} 
S timulated Brillouin scattering (SBS) ${ }^{1,2}$ was considered for many years as an optical gain mechanism for lasers ${ }^{3}$ and for nonlinear optics including phase conjugation ${ }^{4}$ and slow light ${ }^{5}$. Subsequently, the platforms for Brillouin scattering were extended from bulk materials and fibres, to droplets ${ }^{6}$, nanospheres $^{7}$, photonic-crystal fibres ${ }^{8}$ and crystalline resonators ${ }^{9,10}$. The recent demonstration of Brillouin scattering in microspheres ${ }^{11}$ was followed by the Brillouin cooling ${ }^{12}$ and excitation $^{10,13}$ of their vibrational modes as well; which indicates that Brillouin effects can serve as a general mechanism for actuating (and interrogating) vibration in various types of micromechanical resonators. In separate research on optofluidic devices $^{14,15}$, the motion of liquids has been used to control light, but light has rarely been used to actuate a fluid ${ }^{16}$.

In this study, we use the Brillouin scattering of light from sound to excite and measure vibration in a microfluidic optomechanical $(\mu \mathrm{FOM})$ resonator. We design, fabricate and actuate $\mu$ FOM resonators that exhibit mechanical deformation at their solid-fluid interface. Here confinement of the test fluid inside the device mitigates acoustic loss. Conversely, if an optomechanical resonator will just naively be submerged in a liquid, acoustic radiative losses will increase on account of the acoustic impedance of liquids being much larger than that of air.

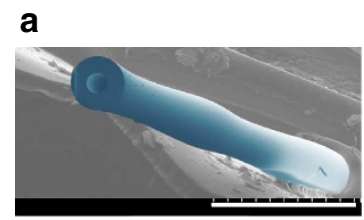

b

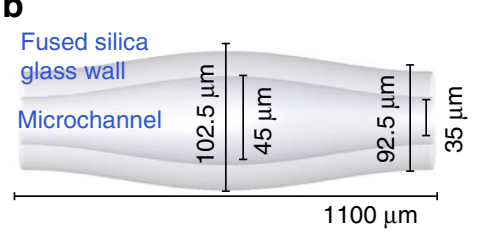

C

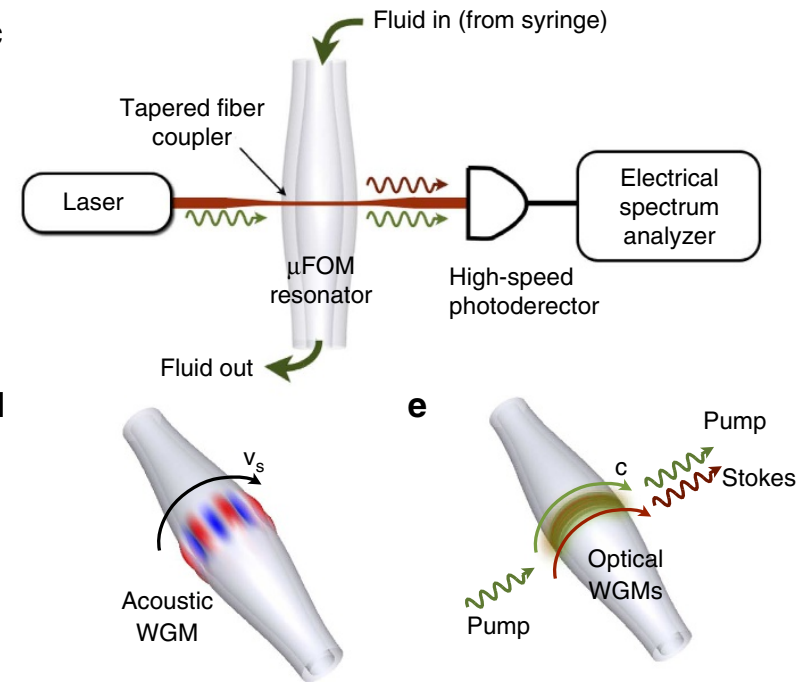

Figure 1 | Overview of the experiment. (a) Colourized scanning electron micrograph of a fused-silica $\mu \mathrm{FOM}$ device, showing the modulation of the radius as a function of position. Scale bar, $200 \mu \mathrm{m}$. (b) Dimensions of the device used to obtain mechanical WGM in Fig. 3b-f. Not drawn to scale. (c) A tapered optical fibre is used to couple light in and out of the whispering-gallery optical modes of the $\mu \mathrm{FOM}$ resonator (without contact). We employ a telecom wavelength $1.5 \mu \mathrm{m}$ pump laser without any modulation. A high-speed photodetector measures the mechanical vibration by means of its optical signature, that is, the beat note between the optical pump and the Stokes-scattered light. The experimental configuration for forward scattering is illustrated here. (d) Illustration of the mechanical WGMs on the $\mu \mathrm{FOM}$ resonator, showing that mechanical displacement is concentrated at the equator. The mode circulates at the velocity of sound, $v_{s}(\mathbf{e})$ Illustration of the optical WGMs of the $\mu \mathrm{FOM}$ resonator that travel at the speed of light, $c$.

\section{Results}

Experimental device and excitation mechanisms. Our $\mu \mathrm{FOM}$ device (Fig. 1a,b) is fabricated from a lengthwise-drawn fusedsilica capillary ${ }^{17}$ with its radius modulated as a function of length (see Methods). At its widest point, this glass device forms a whispering-gallery mode (WGM) microresonator ${ }^{18}$ that resonantly enhances both light and sound (Fig. 1d,e) while supporting a considerable overlap between the optical and mechanical modes. The acoustic modes are optically excited by means of forward ${ }^{10,12,13}$ and backward ${ }^{9,11}$ SBS, that is, F-SBS and B-SBS, respectively.

In the case of $\mathrm{B}-\mathrm{SBS}^{19}$, the incoming pump light (frequency $\left.\omega_{\mathrm{p}}\right)$ photoelastically back-scatters from a high-frequency acoustic wave (frequency $\Omega$ ) in the material to generate a lower frequency Stokes optical signal (frequency $\omega_{\mathrm{s}}=\omega_{\mathrm{p}}-\Omega$ ) as shown in Fig. 2 . Simultaneously, electrostrictive pressure is generated by the two optical signals, which amplifies the acoustic wave. Optical spectrum analysis reveals the optical pump and Stokes signals, as shown previously in Tomes and Carmon ${ }^{11}$. The resulting beat note between pump and Stokes signals measured on an electrical spectrum analyser is representative of the high-frequency acoustic wave. This B-SBS-positive feedback process occurs near $\Omega=11 \mathrm{GHz}$ mechanical frequency in silica when $1.5 \mu \mathrm{m}$ pump light is used ${ }^{19}$. An experimental example of such a mode in this study is shown in Fig. 3f.

The case for forward scattering, that is, F-SBS, is identical to what is described above, except that the pump light photoelastically scatters in the forward direction such that the Stokes signal copropagates with the pump. This reversal of the scattering direction (to forwards) suggests that much smaller phonon momentum is required to bridge between the pump and Stokes momentum gap (Fig. 2). As a result, lower acoustic frequencies (sub-GHz) are more likely for the F-SBS process. Additional details on the energy and momentum conservation requirements ${ }^{20}$ for F-SBS and B-SBS in a circular resonator are discussed

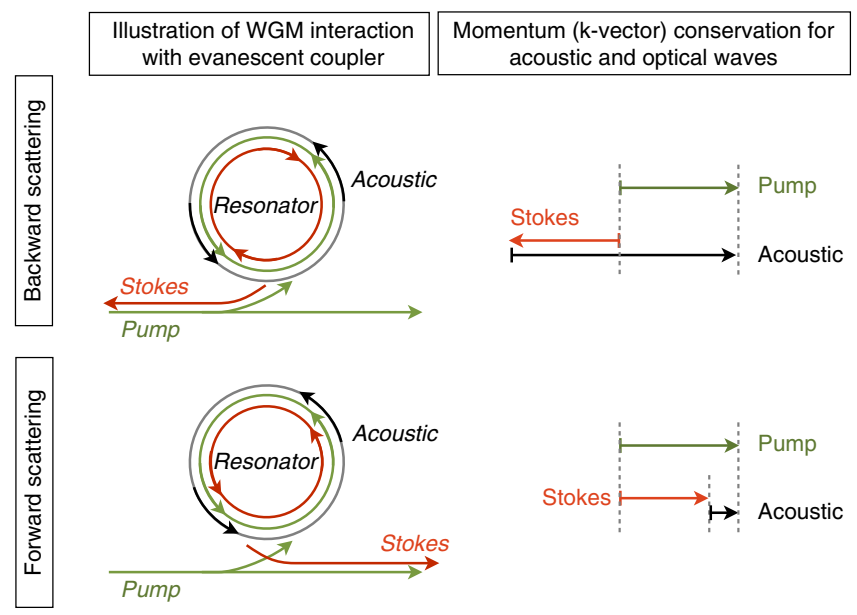

Figure 2 | Acoustic WGM frequencies are determined by momentum conservation. Conservation of momentum between photons and phonons dictates the acoustic WGM frequencies that are generated through SBS. Light travelling in the tapered waveguide evanescently couples to optical WGMs of the resonator, and is scattered to Stokes frequencies in either the forward or backward direction. In the case of back-scattering (B-SBS) momentum conservation enforces long acoustic k-vectors such that the acoustic modes are at high frequencies (that is, $10 \mathrm{GHz}$ regime). Stokes light is received from the same tapered coupler in the backward direction. In forward scattering (F-SBS) momentum, conservation enforces that the acoustic modes are at much lower frequencies (that is, $<1 \mathrm{GHz}$ regime). Stokes light is received in the forward direction as illustrated in Fig. 1c. 
a

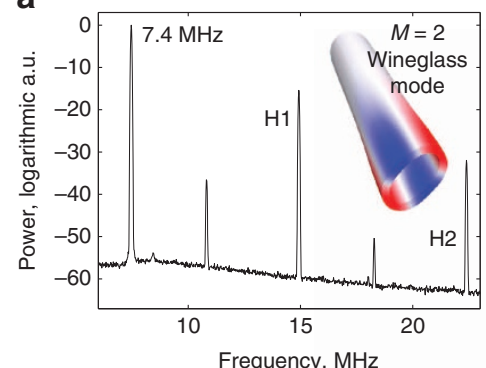

d

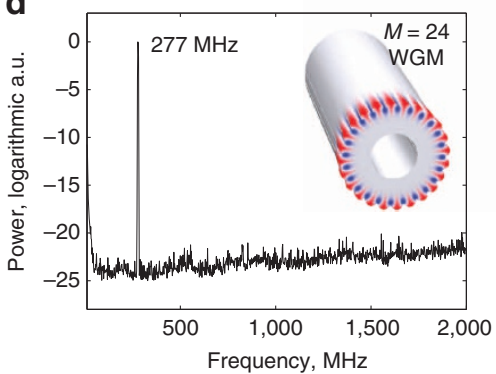

b
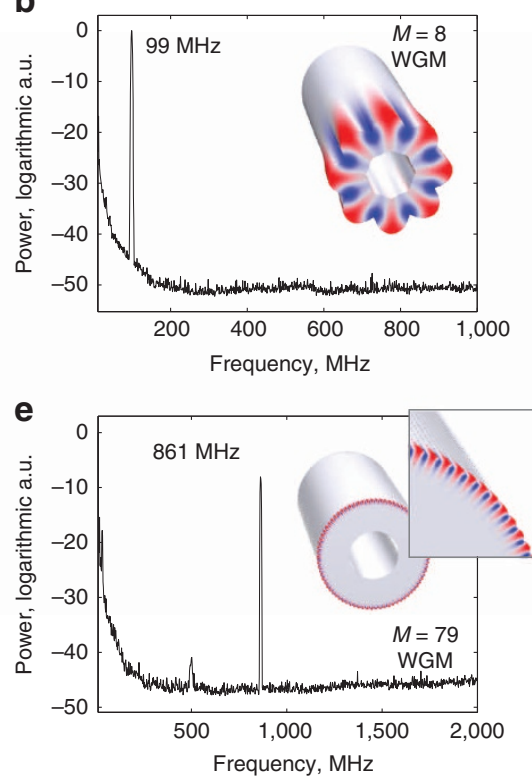

C

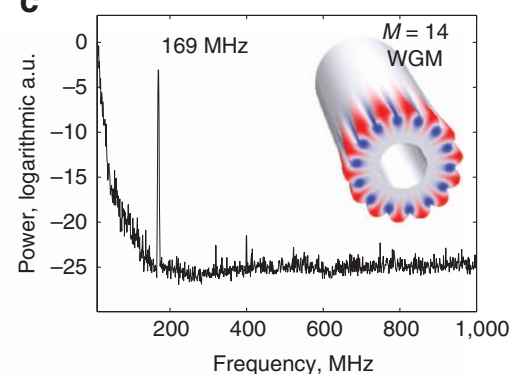

f

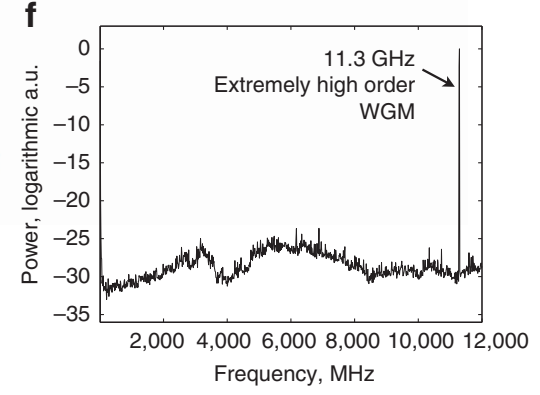

Figure 3 | Experimental observation of mechanical oscillations in water-filled devices. (a) Wineglass mechanical mode oscillation at $7.4 \mathrm{MHz}$. $\mathrm{H} 1$ and $\mathrm{H} 2$ are the first and second harmonics, respectively. Peaks at 10.81 and $18.27 \mathrm{MHz}$ are caused by other mechanical modes of vibration being actuated. (b-e) Acoustic WGM oscillations of various azimuthal mode orders identified with the number $M$. These modes are excited by forward SBS. (f) Extremely high-order acoustic WGM oscillations near $11 \mathrm{GHz}$ are excited by backward SBS. a.u., arbitrary unit.

in $^{9-13,21-23}$. Specifically for the capillary geometry that we use here, it is indicated ${ }^{24}$ that a wide variety of acoustic WGMs exist in the shell-type geometry.

Excitation of acoustic WGMs. We experimentally excite acoustic WGM oscillations in a water-filled $\mu \mathrm{FOM}$ resonator, ranging in frequency from 99 to $11,000 \mathrm{MHz}$ (Fig. 3b-f). As described in Fig. 2, the 'Brillouin' optomechanical process ${ }^{13}$ allows for excitation and measurement of these acoustic WGM oscillations by coupling continuous-in-time light at an optical resonance, while interrogating the optomechanical oscillation at the fibre output of the system (see Methods). No modulation of the pump laser is needed. We introduce and extract light from the optical WGMs by means of evanescent coupling with a tapered optical fibre ${ }^{25,26}$ (as shown in Fig. 1c). Optical ring-down measurements ${ }^{27}$ indicate optical quality factors in excess of $Q_{\mathrm{o}}=10^{8}$ (highest measured $Q_{\mathrm{o}}=1.6 \times 10^{8}$ ). No contact between the fibre and the $\mu \mathrm{FOM}$ resonator is required for mechanical transduction, so the acoustic quality factors are not degraded. The same optical fibre taper is used to extract the Stokes scattered light from the device, which is then used as a measure of the mechanical vibration within the structure ${ }^{12}$. All of our experiments are performed at room temperature and atmospheric pressure, and various solutions are introduced into the device by means of a syringe pump.

We calculate the mechanical modes of the $\mu$ FOM resonator using three-dimentional finite element modelling using Comsol Multiphysics (http://www.comsol.com/) as described previously in Zehnpfennig et al. ${ }^{22}$ and Bahl et al. ${ }^{24}$ Only the glass capillary is modelled, without a liquid, to extract the mechanical modes of the device that correspond to the observed oscillation. Each of these modes is identified with a M-number which is the azimuthal mode order quantifying the number of acoustic wavelengths around the equatorial circumference of the device. In addition, a $M=2 \mathrm{WGM}$ at $7 \mathrm{MHz}$ is observed (Fig. 3a), that is generally referred to as a wineglass mode. The harmonics in this wineglass mode oscillation (H1, H2 in Fig. 3a) are typical for such a
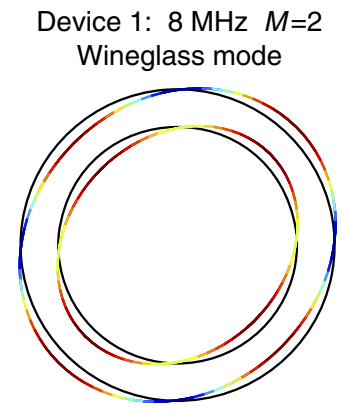

b

Device 2: $99 \mathrm{MHz} \quad M=8$ Whispering-gallery mode

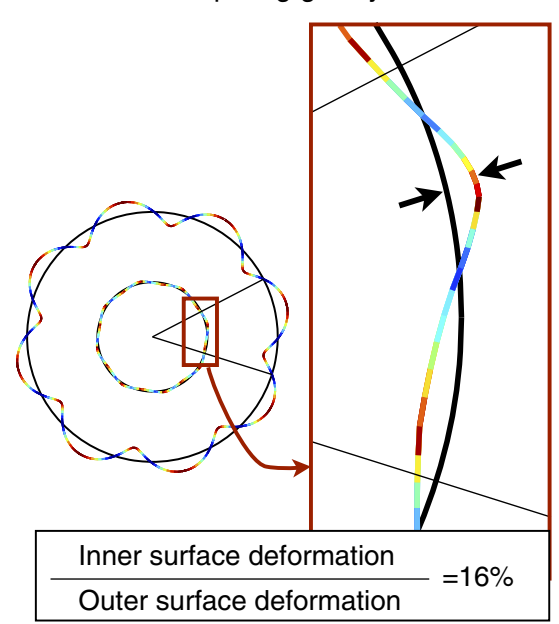

Figure 4 | Calculated equatorial mechanical mode profiles. (a) For the $M=2$ wineglass mode at $8 \mathrm{MHz}$ (experimentally appears at $7.4 \mathrm{MHz}$, see Fig. 3a) the displacement ratio of internal and external surfaces is almost 1:1 at the equator. (b) For the experimentally observed $M=8$ acoustic WGM at $99 \mathrm{MHz}$ (see Fig. 3b), the inner surface experiences $16 \%$ displacement relative to the outer surface. 
low frequency modes and result from the fact that this is a standing-wave type vibration excited by centrifugal radiation pressure $^{28-31}$, as opposed to the travelling-wave vibrations in Fig. $3 b-f$.

Quantifying solid-liquid interaction. The penetration of sound to the liquid scales with the ratio between the acoustic wavelength and the thickness of the resonator wall. Therefore, the $7.4 \mathrm{MHz}$ $(\mathrm{M}=2)$ mode is calculated to have large penetration into the fluid (Fig. 4a). Finite element calculation shows that the $99 \mathrm{MHz}$ $(\mathrm{M}=8)$ mode has a $16 \%$ penetration to fluid, defined by the deformation amplitude at the inner fluid interface divided by the maximum deformation (Fig. 4b). An arguably better method of quantifying the efficiency of interaction with the liquid would be to calculate the ratio of energy stored in fluid deformation to the energy stored in the glass deformation. As this requires modelling of the fluid as well, this preferred quantification method is not chosen here. The higher $M$ modes have lower penetration into the liquid. However, as we can fabricate the resonator with walls as thin as $560 \mathrm{~nm}$ (ref. 32) we believe that a large acoustic penetration to water will be possible in such thin-wall silica bubble resonators even for the $11 \mathrm{GHz}$ mode $(M \approx 650)$, which can open a rare hypersonic window for the acoustic analysis of liquids. Reduction in wall thickness may even allow access to an interesting regime where giant-enhanced Brillouin scattering is predicted $^{33}$.

Characterization of mechanical mode. We measure that the minimum power required to excite these oscillations in the waterfilled $\mu$ FOM device is $158 \mu \mathrm{W}$ (Fig. 5a). We also measured a mechanical quality factor $Q_{\mathrm{m}}=4,700$ for the $99 \mathrm{MHz}(M=8)$ mode in this water-filled device (Fig. 5b), via the sub-threshold oscillation linewidth, which implies $Q_{\mathrm{m}} \times$ Frequency $=4.6 \times 10^{11}$. This 'f $\mathrm{Q}$ product' compares well against the previously measured $Q_{\mathrm{m}}=12,300$ in a solid silica sphere for a $95-\mathrm{MHz}$ mode ${ }^{12}$.

This $99 \mathrm{MHz}(M=8)$ mode of vibration can be maintained as long as the CW input laser power is provided. A spectrogram demonstrating this stability (Fig. 5c) is obtained over 140 seconds
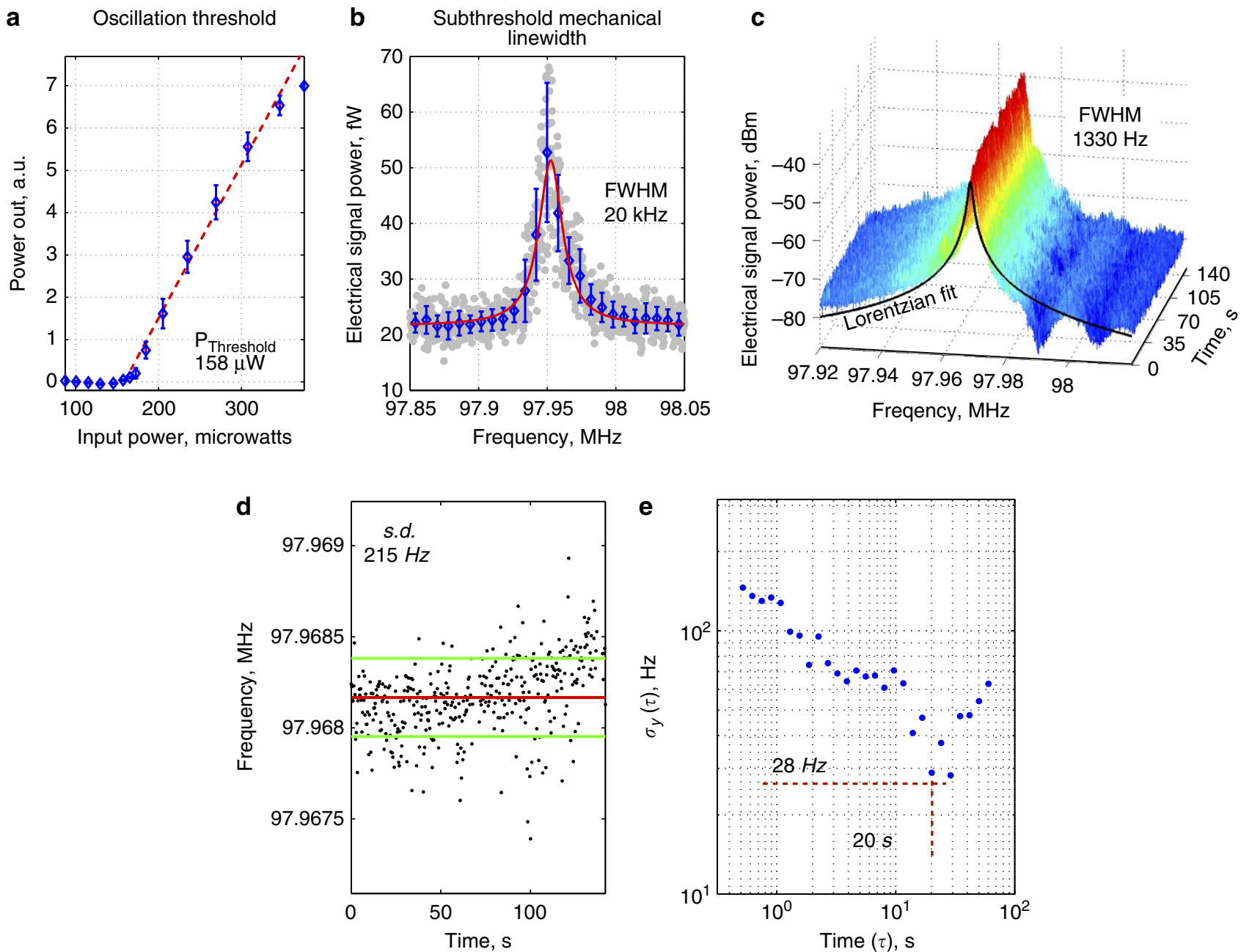

Figure 5 | Characterization of $\mathbf{9 9} \mathbf{~ M H z}$ acoustic WGM in a water-filled device. (a) Lowest observed optical threshold power to excite mechanical oscillation is $158 \mu \mathrm{W}$. Error bars indicate variance of each averaged measurement. (b) The intrinsic mechanical linewidth (mechanical quality factor) is directly observed at very low input optical power. Error bars indicate local variance of the averaged raw data. (c) A spectrogram of the mechanical oscillation frequency spectrum is obtained with above-threshold input optical power. $x$ axis is mechanical frequency measured through the beat note of the pump and the vibration-scattered Stokes light on a photodetector, $y$ axis is logarithmic mechanical oscillation power, and depth axis is time. In this example, the laser was rapidly scanned back-and-forth through the region of the optical mode that exhibits phase-matching for this mechanical oscillation.

(d) Peak frequencies extracted from spectrogram of $\mathbf{c}$ show a s.d. of $215 \mathrm{~Hz}$. (e) Allan deviation plot of the frequency data in $\mathbf{d}$ provides an estimate of the potential sensor resolution. a.u., arbitrary unit. 


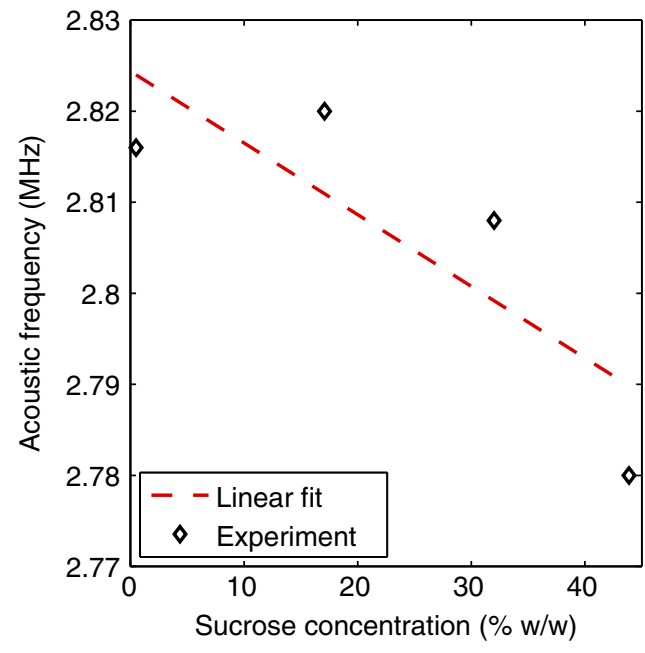

Figure 6 | Optomechanical sensing of sucrose solutions. We experimentally measure the frequency of a $2.8 \mathrm{MHz}$ wineglass acoustic mode $(M=2)$ as the concentration of sucrose solution inside the $\mu \mathrm{FOM}$ resonator is varied. The size of the markers in the figure is approximately the s.d. (better than $1 \mathrm{kHz}$ ) of each frequency measurement. The dashed red line is a linear fit to the data, which indicates a downward trend consistent with increasing effective mass of the resonator. Resonator outer diameter is $184 \mu \mathrm{m}$.

while periodically scanning through the optical resonance that excites this mechanical mode. While such oscillation data is typically captured with the laser at a fixed frequency, we practically found that scanning our pump laser through the optical resonance is more repeatable. This is because scanning is less affected by unknown drifts. We detect the peak oscillation frequencies (Fig. 5d) in this spectrogram by means of Lorentzian curve fits (Fig. 5c) at each time-point. The standard deviation of the peak frequency is calculated to be $215 \mathrm{~Hz}$ along this $140-$ second period.

In order to better characterize the stability of the oscillation and the ability of this system to function as a sensor, we calculate the Allan deviation (square root of the two-point variance) of the frequency data, which charts the frequency deviation as a function of averaging time (Fig. 5e). It is seen that short-term and long-term frequency drifts dominate device performance. For instance, the long-term upward drift in Fig. 5d manifests as an up-turn in the Allan Deviation data (Fig. 5e) for long averaging times. The short-term frequency instabilities manifest as a large deviation for short averaging times. A 'sweet spot' occurs in between, indicating that a 20 -s moving average of the sensor output will give us best resolution.

Operation with high-density high-viscosity liquids. We perform an experiment to prove that the optomechanical interaction can be sustained even when the motional mass is high (that is, highdensity liquid) and when the fluid-related acoustic energy losses are high (that is, high viscosity). We incorporate sucrose (table sugar) into an aqueous solution inside the resonator and measure the sensitivity of the optomechanically actuated wineglass mode to the sucrose concentration of the fluid. Optomechanical oscillation is observed at all tested sucrose concentrations. The resulting relationship between acoustic frequency and the sucrose concentration exhibits a non-monotonic trend, which we plot in Fig. 6 along with a linear fit to the data. The net decreasing frequency trend is consistent with increasing effective mass of the oscillator as the fluid density increases. The visibly non-monotonic nature of the trend, however, might originate from non-perturbative high-sugar concentrations resulting in nonlinear change in frequency. For a complete model one should consider the change in the fluid properties with the sucrose concentration, including the change of speed of sound ${ }^{34}$. We note that at the highest sucrose concentration that we test in this experiment, the solution has a viscosity that is $\sim 3$-times greater than that of blood ${ }^{35}$. The actuation of optomechanical oscillation with such a high-viscosity liquid opens up the possibility for investigating the acoustic properties of viscous bio-analytes.

\section{Discussion}

In this work, we demonstrate a bridge between optomechanics and microfluidics by confining various liquids inside a hollow microfluidic optomechanical resonator. We present experimental evidence that the optomechanical interaction in the $\mu \mathrm{FOM}$ device is dependent on the fluid contained within. These results are, therefore, a step towards novel experiments probing optomechanics on non-solid phases of matter. In particular, the high frequency, high quality-factor mechanical modes demonstrated in this work may enable strongly localized, high-sensitivity, optomechanical interaction with chemical and biological analytes $^{36,37}$ as environmental parameters like pressure and cell nutrients are relatively easy to control inside the hollow resonator $^{32,38,39}$, and as the liquid volume within the device is the scale of a living cell.

In contrast to other optomechanical systems that typically involve standing-wave mechanical vibrations, here circulating phonons comprises the acoustic WGMs ${ }^{13,22}$, which are essentially acoustic vortices ${ }^{40-42}$. These travelling-wave type acoustic modes carry angular momentum, which creates possibility of using $\mu F O M$ devices to enable optomechanical interactions with vortices in various liquids ${ }^{43-45}$ and condensates.

\section{Methods}

Microfluidic device fabrication. The $\mu$ FOM device (Fig. 1a,b) is fabricated with a fused-silica glass capillary that is pulled lengthwise using linear actuators, while being heated with infrared lasers $\left(\mathrm{CO}_{2}\right.$ at $\left.10.6 \mu \mathrm{m}\right)$. By controlling the power of the manufacturing laser in the process of pulling, we can control the device diameter along its length. This process has also previously been developed in Sun et al. ${ }^{17}$ Optical WGMs and acoustic WGMs are simultaneously well-confined in the regions of large diameter sandwiched between regions of narrow diameter (Fig. 1d,e), enabling a high-degree of modal overlap. Therefore, these large diameter regions form the $\mu \mathrm{FOM}$ resonators, and multiple such resonators can be built on a single capillary.

Experimental method. The $\mu \mathrm{FOM}$ resonator is placed in close proximity $(<1 \mu \mathrm{m}$ distance) to tapered optical fibre $e^{25,26}$ (as shown in Fig. 1c), such that evanescent coupling from the taper allows light to be introduced and extracted from the optical WGMs of the device. A CW fibre-coupled tunable $1.5 \mu \mathrm{m}$ laser is used as the source (Newfocus TLB-6328), and a fibre-coupled high-speed photodetector is used to monitor vibration. Mechanical vibration is stimulated within the device by pumping-specific optical resonances where the three-mode phase-match exists. The internally generated Stokes-shifted laser is then also collected by the tapered fibre, and the temporal interference of this new Brillouin laser against the partially transmitted pump laser is used to infer the mechanical response using the photodetector ${ }^{12,13}$. An optical spectrum analyser is used to verify that four-wave mixing and Raman scattering are not responsible for the beat note observed in the electrical spectrum

\section{References}

1. Shen, Y. R. \& Bloembergen, N. Theory of Stimulated Brillouin and Raman scattering. Phys. Rev. 137, A1787-A1805 (1965).

2. Yariv, A. Quantum theory for parametric interactions of light and hypersound. IEEE J. Quant. Electron. 1, 28-36 (1965).

3. Chiao, R. Y., Townes, C. H. \& Stoicheff, B. P. Stimulated Brillouin scattering and coherent generation of intense hypersonic waves. Phys. Rev. Lett. 12, 592-595 (1964)

4. Zel'dovich, B. Y., Popocivhec, V. I., Ragul'skii, V. V. \& Faisullov, F. S. Connection between the wave fronts of the reflected and exciting light in stimulated MandelÕshtam-Brillouin scattering. JETP Lett. 15, 109 (1972) 
5. Okawachi, Y. et al. Tunable all-optical delays via Brillouin slow light in an optical fiber. Phys. Rev. Lett. 94, 153902 (2005).

6. Zhang, J. -Z. \& Chang, R. K. Generation and suppression of stimulated brillouin scattering in single liquid droplets. J. Opt. Soc. Am. B 6, 151-153 (1989).

7. Kuok, M. H., Lim, H. S., Ng, S. C., Liu, N. N. \& Wang, Z. K. Brillouin Study of the quantization of acoustic modes in nanospheres. Phys. Rev. Lett. 90, 255502 (2003).

8. Dainese, P. et al. Stimulated Brillouin scattering from multi-GHz-guided acoustic phonons in nanostructured photonic crystal fibres. Nat. Phys. 2, 388-392 (2006).

9. Grudinin, I. S., Matsko, A. B. \& Maleki, L. Brillouin lasing with a $\mathrm{CaF}_{2}$ whispering gallery mode resonator. Phys. Rev. Lett. 102, 043902 (2009).

10. Savchenkov, A. A., Matsko, A. B., Ilchenko, V. S., Seidel, D. \& Maleki, L. Surface acoustic wave opto-mechanical oscillator and frequency comb generator. Opt. Lett. 36, 3338-3340 (2011).

11. Tomes, M. \& Carmon, T. Photonic micro-electromechanical systems vibrating at X-band (11-GHz) rates. Phys. Rev. Lett. 102, 113601 (2009).

12. Bahl, G., Tomes, M., Marquardt, F. \& Carmon, T. Observation of spontaneous Brillouin cooling. Nat. Phys. 8, 203-207 (2012).

13. Bahl, G., Zehnpfennig, J., Tomes, M. \& Carmon, T. Stimulated optomechanical excitation of surface acoustic waves in a microdevice. Nat. Commun. 2, 403 (2011).

14. Psaltis, D., Quake, S. R. \& Yang, C. Developing optofluidic technology through the fusion of microfluidics and optics. Nature 442, 381-386 (2006).

15. Stolyarov, A. M. et al. Microfluidic directional emission control of an azimuthally polarized radial fibre laser. Nat. Photon. 6, 229-233 (2012).

16. Zhang, J. -Z. \& Chang, R. K. Shape distortion of a single water droplet by laserinduced electrostriction. Opt. Lett. 13, 916-918 (1988).

17. Sun, Y., Shopova, S. I., Wu, C. -S., Arnold, S. \& Fan, X. Bioinspired optofluidic FRET lasers via DNA scaffolds. Proc. Natl Acad. Sci. USA 107, 16039-16042 (2010).

18. Chang, R. K. Optical Processes in Microcavities (World Scientific Publishing Company, 1996).

19. Boyd, R. W. in Nonlinear Optics 3 edn Ch. 9 (Elsevier, 2008).

20. Yariv, A. Quantum Electronics 3 edn (Wiley, 1989).

21. Matsko, A. B., Savchenkov, A. A., Ilchenko, V. S., Seidel, D. \& Maleki, L. Optomechanics with surface-acoustic-wave whispering-gallery modes. Phys Rev. Lett. 103, 257403 (2009).

22. Zehnpfennig, J., Bahl, G., Tomes, M. \& Carmon, T. Surface optomechanics: calculating optically excited acoustical whispering gallery modes in microspheres. Opt. Express 19, 14240-14248 (2011).

23. Shelby, R., Levenson, M. \& Bayer, P. Resolved forward Brillouin scattering in optical fibers. Phys. Rev. Lett. 54, 939-942 (1985).

24. Bahl, G., Fan, X. \& Carmon, T. Acoustic whispering-gallery modes in optomechanical shells. New. J. Phys. 14, 115026 (2012).

25. Knight, J., Cheung, G., Jacques, F. \& Birks, T. Phase-matched excitation of whispering-gallery-mode resonances by a fiber taper. Opt. Lett. 22, 1129-1131 (1997).

26. Cai, M. \& Vahala, K. Highly efficient hybrid fiber taper coupled microsphere laser. Opt. Lett. 26, 884-886 (2001).

27. Savchenkov, A. A., Matsko, A. B., Mohageg, M. \& Maleki, L. Ringdown spectroscopy of stimulated raman scattering in a whispering gallery mode resonator. Opt. Lett. 32, 497-499 (2007)

28. Carmon, T., Rokhsari, H., Yang, L., Kippenberg, T. \& Vahala, K. Temporal behavior of radiation-pressure-induced vibrations of an optical microcavity phonon mode. Phys. Rev. Lett. 94, 223902 (2005).

29. Rokhsari, H., Kippenberg, T., Carmon, T. \& Vahala, K. Radiation-pressuredriven micro-mechanical oscillator. Opt. Express 13, 5293-5301 (2005).
30. Kippenberg, T. J., Rokhsari, H., Carmon, T., Scherer, A. \& Vahala, K. J. Analysis of radiation-pressure induced mechanical oscillation of an optical microcavity. Phys. Rev. Lett. 95, 033901 (2005).

31. Kim, K. H. et al. Cavity optomechanics on a microfluidic resonator with water and viscous liquids. Preprint at http://arxiv.org/abs/1205.5477 (2012).

32. Lee, W. et al. A quasi-droplet optofluidic ring resonator laser using a microbubble. Appl. Phys. Lett. 99, 091102 (2011).

33. Rakich, P. T., Reinke, C., Camacho, R., Davids, P. \& Wang, Z. Giant enhancement of stimulated brillouin scattering in the subwavelength limit. Phys. Rev. 2, 011008 (2012).

34. Rao, M. A., Rizvi, S. S. H. \& Datta, A. K. Engineering Properties of Foods 3 edn (Taylor and Francis, CRC Press, 2005).

35. Instrumentation Specialities Company. Iscotables: A handbook of Data for Biological and Physical Scientists (Instrumentation Specialities Company, 1989).

36. Burg, T. P. et al. Weighing of biomolecules, single cells and single nanoparticles in fluid. Nature 446, 1066-1069 (2007).

37. Barton, R. A. et al. Fabrication of a nanomechanical mass sensor containing a nanofluidic channel. Nano. Lett. 10, 2058-2063 (2010).

38. Sumetsky, M., Dulashko, Y. \& Windeler, R. S. Optical microbubble resonator. Opt. Lett. 35, 898-900 (2010).

39. Berneschi, S. et al. High q silica microbubble resonators fabricated by arc discharge. Opt. Lett. 36, 3521-3523 (2011).

40. Lugt, H. J. Vortex Flow in Nature and Technology (Wiley-Interscience, 1983).

41. Sathaye, A. \& Lal, A. An acoustic vortex generator for microfluidic particle entrapment. IEEE Ultrasonics Symposium 1, 641-644 (2001).

42. Dashti, P. Z., Alhassen, F. \& Lee, H. P. Observation of orbital angular momentum transfer between acoustic and optical vortices in optical fiber. Phys Rev. Lett. 96, 043604 (2006).

43. Feynman, R. in Progress in Low Temperature Physics. Vol 1, Ch. 2, 17-53 (1955).

44. Roberts, P. H. \& Donnelly, R. J. Superfluid mechanics. Annu. Rev. Fluid. Mech. 6, 179-225 (1974)

45. Marston, P. L. \& Fairbank, W. M. Evidence of a large superfluid vortex in ${ }^{4} \mathrm{He}$. Phys. Rev. Lett. 39, 1208-1211 (1977).

\section{Acknowledgements}

This work was supported by the Defense Advanced Research Projects Agency (DARPA) Optical Radiation Cooling and Heating in Integrated Devices (ORCHID) programme and through a grant from the Air Force Office of Scientific Research (AFOSR).

\section{Author contributions}

G.B., K.H.K., X.F. and T.C. designed and conceived the experiments. G.B., K.H.K., W.L. and J.L. designed and developed the fabrication for the microfluidic optomechanical resonators. G.B. and K.H.K. carried out the experiments and numerical calculations. All authors jointly analysed the data, and cowrote the paper. X.F. and T.C. supervised all aspects of this project.

\section{Additional information}

Competing financial interests: The authors declare no competing financial interests.

Reprints and permission information is available online at http://npg.nature.com/ reprintsandpermissions/

How to cite this article: Bahl, G. et al. Brillouin cavity optomechanics with microfluidic devices. Nat. Commun. 4:1994 doi: 10.1038/ncomms2994 (2013). 